\title{
A new method for preparing microporous titanium pillared clays
}

\author{
Jenn-Tsuen Lin, Sung-Jeng Jong and Soofin Cheng* \\ Department of Chemistry, National Taiwan University, Taipei, Taiwan 107
}

(Received 8 December 1992; accepted 2 April 1993)

\begin{abstract}
A new method for reproducible preparation of Ti-pillared montmorillonite with microporous structure, high surface area and high thermal stability is reported. The $<2 \mu \mathrm{m}$ fraction of a physically purified Wyoming $\mathrm{Na}^{+}-\mathrm{Ca}^{2+}$-montmorillonite (commercial designation, Volclay SPV 200) was used as starting material. The pillaring agent was prepared by mixing a $\mathrm{TiCl}_{4}$ /ethanol solution with a solution of glycerin and water. XRD analyses of the pillared clays showed that the as-synthesized sample had a basal spacing of $21.3 \AA$, that shrank to $17.7 \AA$ after calcination at $773 \mathrm{~K}$ and to $17.4 \AA$ after calcination at $973 \mathrm{~K}$. By subtracting the basal thickness of clay, the interlayer spaces of $10.8,8.2$ and $7.9 \AA$ were obtained. These distances implied that $\mathrm{Ti}$ was incorporated into the interlayers in some form of polynuclear clusters. The pillared clays had specific surface areas of 379,365 and $286 \mathrm{~m}^{2} / \mathrm{g}$ after calcination at 573,773 and $973 \mathrm{~K}$, respectively. The $\mathrm{N}_{2}$ adsorption/desorption isotherms indicated that the corresponding samples contained both the micro- and meso-porous structures. The diameters of the micropores were consistent with the interlayer spaces obtained from XRD patterns. The micropores contributed $\sim 88 \%$ to the total pore volume of the sample calcined at $573 \mathrm{~K}$, that was found to be retained after calcination at $773 \mathrm{~K}$ but reduce to $\sim 65 \%$ of its origin after calcination at $973 \mathrm{~K}$. The presence of glycerin in the preparation procedure was found to be essential in order to prepare Ti-pillared clay of high thermal stability.
\end{abstract}

Keywords: titanium oxide; pillared clay; preparation; glycerin; thermal stability

Pillaring layered compounds with bulky inorganic species is a well-known route to prepare microporous materials. The increasing number of studies in this field is stimulated by their potential applications in catalysis and adsorption. Among the available layered compounds, clays have received the most attention, and pillared clays seem to have great potential in the area of acid catalyzed reactions, such as cracking and alkylation [1-3]. Unlike zeolites, which are mainly composed of oxides of $\mathrm{Si}$ and $\mathrm{Al}$, the structure and properties of the pillared assemblies can be modified by varying the chemical components of either the basal layers or the interlayer pillars [4-7]. However, the success in the preparation of specific

* Corresponding author pillared compounds depends very much on a full understanding of the chemistry involved in the pillaring processes.

Titanium oxide has several significant and distinctive properties as a catalyst or catalyst support. The phenomenon of strong metal-support interaction (SMSI) was first reported for noble metals supported on $\mathrm{TiO}_{2}[8]$. $\mathrm{TiO}_{2}$ based catalysts were found to be the best catalysts for the selective reduction of $\mathrm{NO}_{x}$ with $\mathrm{NH}_{3}$ [9], and for xylene oxidation to phthalic anhydride $[10,11]$. Moreover, titanium oxide is a typical photocatalyst and is responsible for a variety of organic reactions [12]. In a recent article, Yoneyama and coworkers [13] reported that the photocatalytic activity of montmorillonite pillared with $\mathrm{TiO}_{2}$ clusters was higher than the $\mathrm{TiO}_{2}$ powder in decomposition of 2-pro- 
panol and carboxylic acids (2-9 carbon atoms in the chain).

In spite of their promising applications, only a few preparation methods for titanium pillared clays have been published so far. From these publications, it is known that the synthesis conditions are critical, primarily due to the diversity of titanium species formed in aqueous solutions. In 1986, Sterte [14] reported for the first time on the preparation of Ti-pillared clays. A $\mathrm{TiCl}_{4} / \mathrm{HCl}$ solution was used as the pillaring agent. The basal spacing of the products heated at temperatures above $473 \mathrm{~K}$ determined to be about $28 \AA$ by X-ray powder diffraction and $\mathbf{N}_{2}$ adsorption/desorption isotherms. Later, Bernier et al. [15] performed a detailed study on the experimental conditions with the same pillaring agent. The synthesis conditions were found to be critical with respect to the morphology and texture of the final product. The basal spacing of the uncalcined samples could be varied from 24.9 to $13.8 \AA$. A different method was reported by Yamanaka et al. [16,17], who used titanium oxide sol as the pillaring agent which was prepared by hydrolysis of titanium tetraisopropyloxide followed by peptization with $\mathrm{HCl}$. The resulting products had a basal spacing of $\sim 27 \AA$. The pore size was found to correlate with the size of the sol particles, which was in turn dependent on the peptization condition. The third method reported in the literature uses a trinuclear acetatochlorohydroxo titanium(III) complex as the titanium source [18]. The latter is hydrolyzed, oxidized and condensed into polynuclear forms during the pillaring processes. The resulting compounds have a basal spacing around $22 \AA$, but the specific surface area is only $\sim 120 \mathrm{~m}^{2} / \mathrm{g}$. This is much smaller than $\sim 300 \mathrm{~m}^{2} / \mathrm{g}$ for those samples obtained by the previous two methods. Here we report on a new method for preparing Ti-pillared montmorillonite with microporous structure, high surface area and high thermal stability.

A Wyoming $\mathrm{Na}^{+}-\mathrm{Ca}^{2+}$-montmorillonite (commercial designation, Volclay SPV 200) was obtained from the American Colloid Company. Impurity quartz was removed by conventional sedimentation techniques. The fraction with a particle size below $2 \mu \mathrm{m}$ was used as starting material. The cation-exchange capacity of the montmorillon- ite was determined to be $0.83 \mathrm{meq} / \mathrm{g}$. The pillaring agent was prepared by first mixing $\mathrm{TiCl}_{4}$ with twice the amount of ethanol and stirring until homogeneous. $2-8 \mathrm{ml}$ of the partially hydrolyzed titaniumethoxide solution was then added to a $20 \mathrm{ml}$ solution consisting of glycerin and water in 1:1 volumetric ratio and stirred for $3 \mathrm{~h}$. The clear mixture was then added dropwise to one gram of clay dispersed in $100 \mathrm{ml}$ of deionized water. The $\mathrm{pH}$ of the final solution was measured to be 0.44 . After stirring for $4 \mathrm{~h}$, the clay was filtered and washed thoroughly with deionized water. The $\mathrm{pH}$ of the filtrate was $\sim 1.5$.

XRD analyses of the pillared clays were performed on oriented samples prepared by spreading $\sim 0.5 \mathrm{ml}$ of a water suspension of the sample onto a quartz slide, followed by drying at room temperature or calcination at elevated temperatures. XRD patterns were obtained on a Philips PW 1840 automated powder diffractometer, using $\mathrm{Ni}$-filtered $\mathrm{Cu} K \alpha$ radiation. Fig. 1 shows the typical patterns of the Ti-pillared clays prepared by this new method. The basal spacings of these layered compounds were determined from the 001 reflection, which appeared as the strongest peaks in the XRD patterns. The as-synthesized sample has a basal spacing of $21.3 \AA$ which shrinks to $17.7 \AA$ after calcination at $773 \mathrm{~K}$ and further to $17.4 \AA$ after calcination at $973 \mathrm{~K}$. The second strong reflection appearing at $10.9 \AA$ in Fig. 1(a) is likely to correspond to the 002 reflection. By subtracting the basal thickness of the clay of $9.5 \AA$, the interlayer distance is $11.8,8.2$ and $7.9 \AA$, respectively. These distances imply that $\mathrm{Ti}$ is incorporated into the interlayers in the form of polynuclear clusters. Moreover, the pillared clays have specific surface areas of 379,365 and $286 \mathrm{~m}^{2} / \mathrm{g}$ after calcination at 573,773 and $973 \mathrm{~K}$, respectively. Fig. 2 shows the $\mathrm{N}_{2}$ adsorption/desorption isotherms of the corresponding samples. The steep increase in adsorbed volume at low partial pressure is attributed to microporous (pore radius $<10 \AA$ ) condensation, while the hysteresis occurring at high partial pressures is attributed to the mesoporous structures (pore radius of 18-200 $\AA$ ) [19]. The diameters of the micropores are consistent with the interlayer space obtained from the XRD patterns. Therefore, the micropores are most probably generated by 


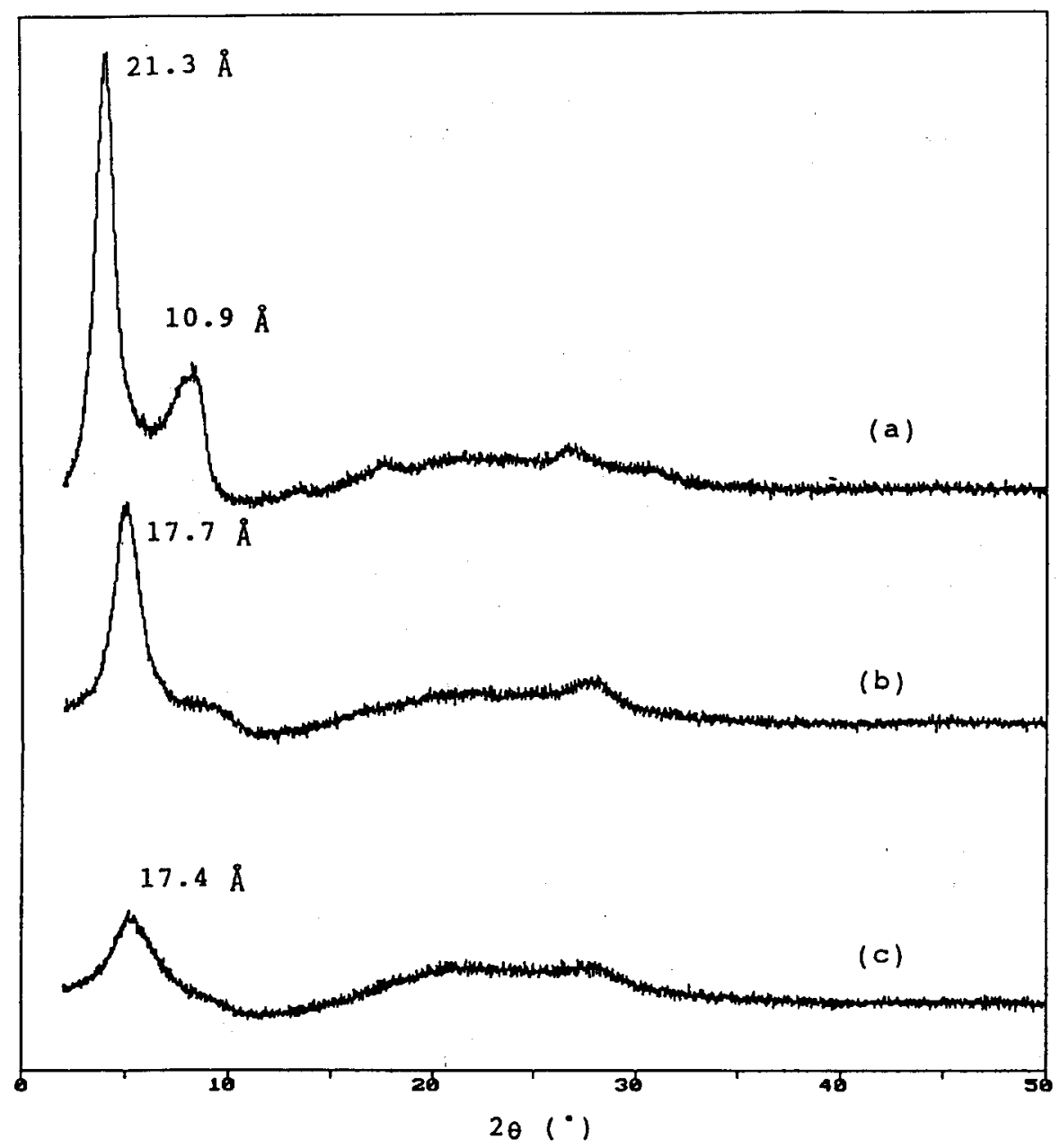

Fig. 1. XRD patterns of Ti-pillared montmorillonite at (a) room temperature and after calcination at (b) $773 \mathrm{~K}$ and (c) $973 \mathrm{~K}$.

the free space in between the adjacent layers, which are propped by the pillars. For the sample calcined at $573 \mathrm{~K}$, the micropores contribute $\sim 88 \%$ to the total pore volume. After calcination at $773 \mathrm{~K}$, the volume of micropores is retained while that of the mesopores increases. The latter is probably due to the void space remaining after the complete combustion of the organic species. When the calcination temperature is further increased to $973 \mathrm{~K}$ the volume of the micropores is reduced to $\sim 65 \%$ of its original value while that of the mesopores is almost unchanged. Judging from the results of XRD analyses, the partial loss of micropores might be due to a severe distortion of the lamellar structure so that the pores in the interior portion of the particles are not accessible by $\mathrm{N}_{2}$ molecules. Nevertheless, the pillared structure is retained after calcination at $973 \mathrm{~K}$ and the micropores still contribute $\sim 70 \%$ to the total pore volume.

The role of glycerin in the pillaring process is not clear at this moment. However, a comparative experiment performed in the absence of glycerin resulted in a pillared clay which is thermally not stable. A detailed study on the nature of the polynuclear titanium species formed in the pillaring agent should result in a deeper insight into the processes occurring during the pillaring step. 


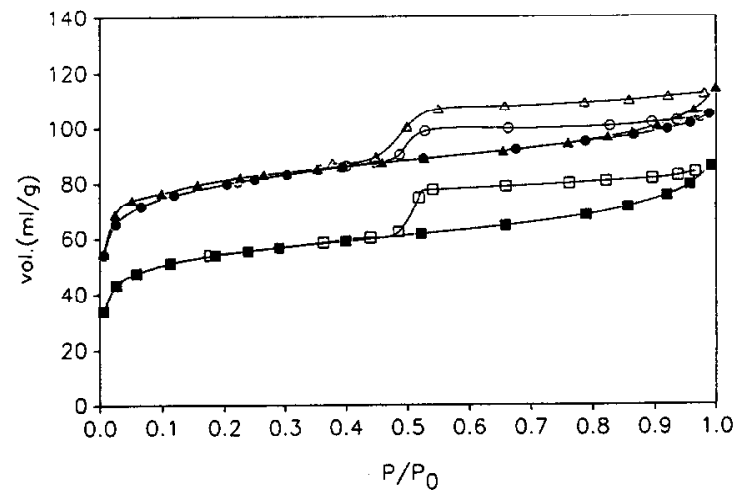

Fig. 2. $\mathrm{N}_{2}$ adsorption/desorption isotherms of Ti-pillared montmorillonite after calcination at $(0 ; 0) 573 \mathrm{~K},(\Delta ; \Delta)$ $773 \mathrm{~K}$ and ( $\mathbf{\square} ; \square) 973 \mathrm{~K}$. (Full symbols correspond to isotherms obtained in the adsorption mode; open symbols correspond to the desorption mode).

\section{Acknowledgements}

Financial support from the National Science Council of the Republic of China is gratefully acknowledged.

\section{References}

1 F. Figueras, Catal. Rev.- Sci. Eng., 30 (1988) 457.

2 T. Matsuda, M. Matsukata, E. Kikuchi and Y. Morita, Appl. Catal., 21 (1986) 297.

3 M.L. Occelli, Stud. Surf. Sci. Catal., 35 (1988) 101.

4 M.A. Drezdon, Inorg. Chem., 27 (1988) 4628.

5 T.J. Pinnavaia, T. Kwon and G.A. Tsigdinos, J. Am. Chem. Soc., 110 (1988) 3653.

6 G. Cao, M.E. Garcia, M. Alcala, L.F. Burgess and T.E. Mallouk, J. Am. Chem. Soc., 114 (1992) 7574.

7 S.-T. Wong and S. Cheng, Inorg. Chem., 31 (1992) 1165.

8 S. Tauster, S.C. Fung and R.L. Garten, J. Am. Chem. Soc., 110 (1978) 170.

9 S. Matsuda, M. Takeuchi, T. Hishinuma, F. Nakajima, T. Narita, Y. Watanabe and M.J. Imanati, Air Pollut. Control Assoc., 28 (1978) 350.

10 A. Vejux and P. Courtine, J. Solid State Chem., 23 (1978) 93.

11 M. Gasior and T. Machey, J. Catal., 83 (1983) 472.

12 I. Izumi, W.W. Dunn, K.O. Wilboun, F.F. Fan and A.J. Bard, J. Phys. Chem., 84 (1980) 3207.

13 H. Yoneyama, S. Haga and S. Yamanaka, J. Phys. Chem., 93 (1989) 4833.

14 J. Sterte, Clays Clay Miner, 34 (1986) 658.

15 A. Bernier, L.F. Admaiai and P. Grange, Appl. Catal., 77 (1991) 269.

16 S. Yamanaka, T. Nishihara, M. Hattori and Y. Suzuki, Mat. Chem. Phys., 17 (1987) 87.

17 S. Yamanaka and M. Hattori, Stud. Surf. Sci. Catal., 60 (1991) 89.

18 T. Kijima, H. Nakazawa and S. Takenouchi, Bull. Chem. Soc. Jpn., 64 (1991) 1395.

19 C.N. Satterfield, Heterogenous Catalysis in Industrial Practice, McGraw-Hill, New York, 1991, p. 39. 